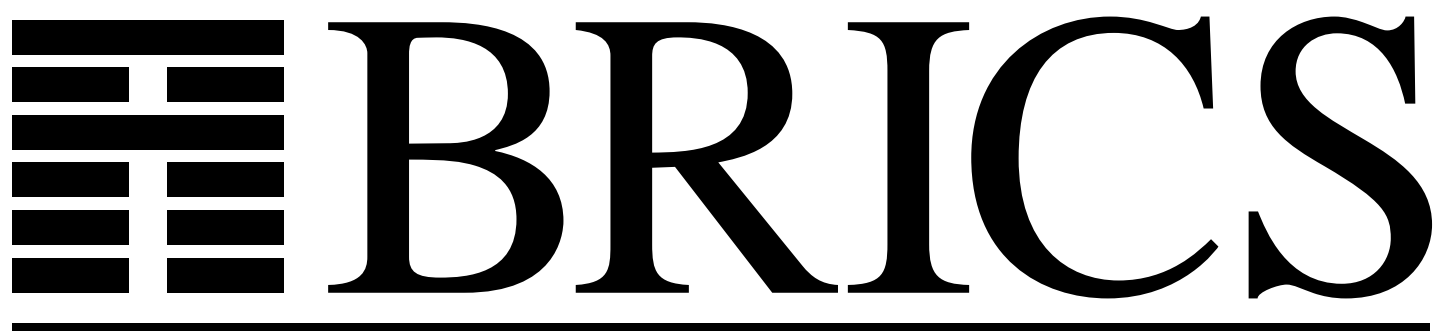

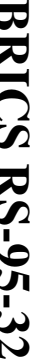

Basic Research in Computer Science

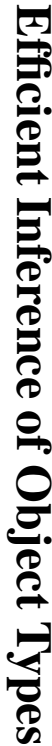

Jens Palsberg 
Copyright (c) 1995, BRICS, Department of Computer Science University of Aarhus. All rights reserved.

Reproduction of all or part of this work is permitted for educational or research use on condition that this copyright notice is included in any copy.

See back inner page for a list of recent publications in the BRICS Report Series. Copies may be obtained by contacting:

\section{BRICS}

Department of Computer Science

University of Aarhus

Ny Munkegade, building 540

DK - 8000 Aarhus C

Denmark

Telephone: +4589423360

Telefax: $\quad+4589423255$

Internet: BRICS@brics.dk

BRICS publications are in general accessible through WWW and anonymous FTP:

htt p: / / unww bri cs. dk/

ftp ftp. bri cs. dk (cd pub/ BR CS) 


\title{
Efficient Inference of Object Types
}

\author{
Jens Palsberg \\ BRICS* \\ Department of Computer Science \\ University of Aarhus \\ Ny Munkegade \\ DK-8000 Aarhus C, Denmark
}

\begin{abstract}
Abadi and Cardelli have recently investigated a calculus of objects [2]. The calculus supports a key feature of object-oriented languages: an object can be emulated by another object that has more refined methods. Abadi and Cardelli presented four first-order type systems for the calculus. The simplest one is based on finite types and no subtyping, and the most powerful one has both recursive types and subtyping. Open until now is the question of type inference, and in the presence of subtyping "the absence of minimum typings poses practical problems for type inference" [2].

In this paper we give an $O\left(n^{3}\right)$ algorithm for each of the four type inference problems and we prove that all the problems are P-complete. We also indicate how to modify the algorithms to handle functions and records.
\end{abstract}

\section{Introduction}

Abadi and Cardelli have recently investigated a calculus of objects [2]. The calculus supports a key feature of object-oriented languages: an object can

\footnotetext{
*Basic Research in Computer Science, Centre of the Danish National Research Foundation. E-mail: palsberg@daimi.aau.dk.
} 
be emulated by another object that has more refined methods. For example, if the method invocation a.l is meaningful for an object $a$, then it will also be meaningful for objects with more methods than $a$, and for objects with more refined methods. This phenomenon is called subsumption.

The calculus contains four constructions: variables, objects, method invocation, and method override. It is similar to the calculus of Mitchell, Honsell, and Fisher [20] in allowing method override, but it differs significantly in also allowing subsumption but not allowing objects to be extended with more methods.

\begin{tabular}{|l|c|c|}
\hline & Abadi, Cardelli [2] & Mitchell, Honsell, Fisher [20] \\
\hline Objects & $\sqrt{ }$ & $\sqrt{ }$ \\
\hline Method override & $\sqrt{ }$ & $\sqrt{ }$ \\
\hline Subsumption & $\sqrt{ }$ & $\sqrt{ }$ \\
\hline Object extension & & \\
\hline
\end{tabular}

Abadi and Cardelli presented four first-order type systems for their calculus. The simplest one is based on finite types and no subtyping, and the most powerful one has both recursive types and subtyping. The latter can type many intriguing object-oriented programs, including objects whose methods return an updated self [2], see also [4, 3, 1].

Open until now is the question of type inference:

Given an untyped program $a$, is a typable? If so, annotate it.

In the presence of subtyping "the absence of minimum typings poses practical problems for type inference" [2].

In this paper we give an $O\left(n^{3}\right)$ algorithm for each of the four type inference problems and we prove that all the problems are $\mathrm{P}$-complete.

Choose: finite types or recursive types.

Choose: subtyping or no subtyping.

In any case: type inference is

P-complete and computable in $O\left(n^{3}\right)$.

Our results have practical significance:

1. For object-oriented languages based on method override and subsumption, we provide the core of efficient type inference algorithms. 
2. The P-completeness indicates that there are no fast NC-class parallel algorithms for the type inference problems, unless $\mathrm{NC}=\mathrm{P}$.

In Section 2 we present Abadi and Cardelli's calculus. For readability, Section 3-6 concentrate on the most powerful of the type systems, the one with recursive types and subtyping. The other type systems requires similar developments that will be summarized in Section 7. We first present the type system (Section 3), and we then prove that the type inference problem is log space equivalent to a constraint problem (Section 4) and a graph problem (Section 5), and we prove that a program is typable if and only if the corresponding graph problem involves a well-formed graph (Section 6). If the graph is well-formed, then a certain finite automaton represents a canonical typing of the program. In Section 7 we give algorithms for all four type inference problems and in Section 8 we prove that all the problems are P-complete under log space reductions. In Section 9 we give three examples of how the most powerful of our type inference algorithms works. Finally, in Section 10 we discuss related work and possible extensions. The reader is encouraged to refer to the examples while reading the other sections.

Our approach to type inference is related to that of Kozen, Schwartzbach, and the present author in [17]. Although the problems that we solve here are much different from that solved in [17], the two approaches have the same ingredients: constraints, graphs, and automata.

We have produced a prototype implementation in Scheme of the most powerful of our type inference algorithms. Experiments have been carried out on a SPARCserver 1000 (with four SuperSPARC processors) running $\mathrm{Scm}$ version $4 \mathrm{e} 1$. For example, the implementation used 24 seconds to process a 58 lines program. This is encouraging because we used a rather slow implementation language and because we did not fine-tune the implementation.

A potential obstacle for practical use of our algorithms is the property that the canonical typing of a program may have a representation of a size which is quadratic in the size of the program. Another potential obstacle may be the use of adjacency matrices to represent certain graphs. If those graphs are sparse in practice, then it may be worthwhile using less spacedemanding data structures at the cost of slower worst-case performance. Further experiments are needed to evaluate the speed and space-usage of the algorithms on programs of realistic size. 


\section{Abadi and Cardelli's Calculus}

Abadi and Cardelli first present an untyped object calculus, called the $\varsigma$ calculus. The s-terms are generated by the following grammar:

$$
\begin{aligned}
a::= & x & & \text { variable } \\
& {\left[l_{i}=\varsigma\left(x_{i}\right) b_{i}{ }^{i \in 1 . . n}\right] } & \left(l_{i} \text { distinct }\right) & \text { object } \\
& a . l & & \text { field selection / method invocation } \\
& a . l \Leftarrow \varsigma(x) b & & \text { field update / method override }
\end{aligned}
$$

We use $a, b, c$ to range over $\varsigma$-terms. An object $\left[l_{i}=\varsigma\left(x_{i}\right) b_{i}{ }^{i \in 1 . . n}\right]$ has method names $l_{i}$ and methods $\varsigma\left(x_{i}\right) b_{i}$. The order of the components does not matter. In a method $\varsigma(x) b$, we have that $x$ is the self variable and $b$ is the body. Thus, in the body of a method we can refer to any enclosing object, like in the Beta language [18].

The reduction rules for $\varsigma$-terms are as follows. If $o \equiv\left[l_{i}=\varsigma\left(x_{i}\right) b_{i}{ }^{i \in 1 . . n}\right]$, then, for $j \in 1 . . n$,

$$
\begin{aligned}
& \text { - o. } l_{j} \leadsto b_{j}\left[o / x_{j}\right] \\
& \text { - o. } l_{j} \Leftarrow \varsigma(y) b \leadsto o\left[l_{j} \leftarrow \varsigma(y) b\right]
\end{aligned}
$$

Here, $a[o / x]$ denotes the $\varsigma$-term $a$ with $o$ substituted for free occurrences of $x$ (after renaming bound variables if necessary); and $o\left[l_{j} \leftarrow \varsigma(y) b\right]$ denotes the $\varsigma$-term $o$ with the $l_{j}$ field replaced by $\varsigma(y) b$. An evaluation context is an expression with one hole. For an evaluation context $a[$.$] , if b \leadsto b^{\prime}$, then $a[b] \leadsto a\left[b^{\prime}\right]$.

A $\varsigma$-term is said to be an error if it is irreducible and it contains either $o . l_{j}$ or $o . l_{j} \Leftarrow \varsigma(y) b$, where $o \equiv\left[l_{i}=\varsigma\left(x_{i}\right) b_{i}{ }^{i \in 1 . . n}\right]$, and $o$ does not contain an $l_{j}$ field.

For examples of reductions, consider first the object $o \equiv[l=\varsigma(x) x . l]$. The expression o.l yields the infinite computation:

$$
o . l \leadsto x . l[o / x] \equiv o . l \leadsto \ldots
$$

As another example, consider the object $o^{\prime} \equiv[l=\varsigma(x) x]$. The method $l$ returns self:

$$
o^{\prime} . l \leadsto x\left[o^{\prime} / x\right] \equiv o^{\prime}
$$


As a final example, consider the object $o^{\prime \prime} \equiv[l=\varsigma(y)(y . l \Leftarrow \varsigma(x) x)]$. The method $l$ returns a modified self:

$$
o^{\prime \prime} . l \leadsto\left(o^{\prime \prime} . l \Leftarrow \varsigma(x) x\right) \leadsto o^{\prime}
$$

These three examples are taken from Abadi and Cardelli's paper [2].

Abadi and Cardelli demonstrate how to encode the pure $\lambda$-calculus in the $\varsigma$-calculus. Note the following difference between these two calculi. In pure $\lambda$-calculus no term yields an error; in the $s$-calculus for example [ ].l yields an error. The reason is that objects are structured values. In a $\lambda$-calculus with pairs, some terms yield errors, like in the s-calculus.

\section{Type Rules}

The following type system for the $\varsigma$-calculus catches errors statically, that is, rejects all programs that may yield errors [2].

An object type is an edge-labeled regular tree. A tree is regular if it has finitely many distinct subtrees. Labels are drawn from some possibly infinite set $\mathcal{N}$ of method names. We represent a type as a non-empty, prefix-closed set of strings over $\mathcal{N}$. One such string represents a path from the root. We use $A, B, \ldots$ to denote types. The set of all types is denoted $T$. A type is finite if it is finite as a set of strings.

For $l_{1}, \ldots, l_{n} \in \mathcal{N}, A_{1}, \ldots, A_{n} \subseteq \mathcal{N}^{*}$ and $\alpha \in \mathcal{N}^{*}$, define

$$
\begin{aligned}
{\left[l_{i}: A_{i}{ }^{i \in 1 . . n}\right] } & =\{\epsilon\} \cup\left\{l_{1} \alpha \mid \alpha \in A_{1}\right\} \cup \ldots \cup\left\{l_{n} \alpha \mid \alpha \in A_{n}\right\} \\
A \downarrow \alpha & =\{\beta \mid \alpha \beta \in A\} .
\end{aligned}
$$

Here, $\left[l_{i}: A_{i}{ }^{i \in 1 . . n}\right]$ is an object type with components $l_{i}: A_{i}$, and $A \downarrow \alpha$ is the subtree of $A$ at $\alpha$ if $\alpha \in A, \emptyset$ if not. The following properties are immediate from the definitions:

(i) $\left[l_{i}: A_{i}{ }^{i \in 1 . . n}\right] \downarrow l_{i}=A_{i}$

(ii) $(A \downarrow \alpha) \downarrow \beta=A \downarrow \alpha \beta$

The set of object types is ordered by the subtyping relation $\leq$ as follows:

$$
A \leq B \quad \text { if and only if } \quad \forall l \in \mathcal{N}: \quad l \in B \Rightarrow(l \in A \wedge A \downarrow l=B \downarrow l)
$$


Clearly, $\leq$ is a partial order. Intuitively, if $A \leq B$, then $A$ may contain more fields than $B$, and for common fields, $A$ and $B$ must have the same type. For example, $[l: A, m: B] \leq[l: A]$, but $[l:[m: A]] \not \leq[l:[]]$. Notice that if $A \leq B$, then $B \subseteq A$.

To state typing rules, Abadi and Cardelli use an explicitly typed version of the $\varsigma$-calculus where each bound variable is annotated with a type.

If $a$ is an explicitly typed $\varsigma$-term, $A$ is an object type, and $E$ is a type environment, that is, a partial function assigning types to variables, then the judgement $E \vdash a: A$ means that $a$ has the type $A$ in the environment $E$. This holds when the judgement is derivable using the following five rules:

$$
\begin{gathered}
E \vdash x: A \quad(\text { provided } E(x)=A) \\
\frac{E\left[x_{i} \leftarrow A\right] \vdash b_{i}: B_{i} \quad \forall i \in 1 . . n}{E \vdash\left[l_{i}=\varsigma\left(x_{i}: A\right) b_{i}{ }^{i \in 1 . . n}\right]: A} \quad\left(\text { where } A=\left[l_{i}: B_{i}{ }^{i \in 1 . . n}\right]\right) \\
\forall j \in 1 . . n: \frac{E \vdash a:\left[l_{i}: B_{i}{ }^{i \in 1 . . n}\right]}{E \vdash a . l_{j}: B_{j}} \\
\forall j \in 1 . . n: \quad \frac{E \vdash a: A \quad E[x \leftarrow A] \vdash b: B_{j}}{E \vdash a . l_{j} \Leftarrow \varsigma(x: A) b: A} \quad\left(\text { where } A=\left[l_{i}: B_{i}{ }^{i \in 1 . . n}\right]\right) \\
\frac{E \vdash a: A \quad A \leq B}{E \vdash a: B}
\end{gathered}
$$

The first four rules express the typing of each of the four constructs in the object calculus and the last rule is the rule of subsumption.

Notice that rule (3) can be replaced by the equivalent rule

$$
\frac{E \vdash a:\left[l_{j}: B_{j}\right]}{E \vdash a \cdot l_{j}: B_{j}}
$$

Notice also that rule (4) can be replaced by the equivalent rule

$$
\frac{E \vdash a: A \quad E[x \leftarrow A] \vdash b: B_{j}}{E \vdash a . l_{j} \Leftarrow \varsigma(x: A) b: A} \quad\left(\text { where } A \leq\left[l_{j}: B_{j}\right]\right)
$$

If $E \vdash a: A$ is derivable, we say that $a$ is well-typed with type $A$. An untyped $\varsigma$-term $a$ is said to be typable if there exists an annotated version of $a$ which is well-typed.

For comparison with the typing rules for simply typed $\lambda$-calculus, notice that: 
- Rule (1) is identical to the rule for variables in $\lambda$-calculus;

- Rule (2) can be understood as the rule for object type introduction, just like the rule for $\lambda$-abstraction is the rule for function type introduction; and

- Rule (3) can be understood as the rule for object type elimination, just like the rule for application is the rule for function type elimination in $\lambda$-calculus.

Rule (4) has no obvious counterpart among the typing rules for simply typed $\lambda$-calculus.

For examples of type derivations, let us consider the three example terms from Section 2. First consider the object $o \equiv[l=\varsigma(x) x . l]$. The expression $o . l$ can be typed as follows, with $x$ implicitly typed with $[l:[]]$ :

$$
\frac{\frac{\emptyset[x \leftarrow[l:[]]] \vdash x:[l:[]]}{\emptyset[x \leftarrow[l:[]]] \vdash x . l:[]}}{\emptyset \vdash o:[l:[]]}
$$

Consider then the object $o^{\prime} \equiv[l=\varsigma(x) x]$. The expression $o^{\prime} . l$ can be typed as follows, with $x$ implicitly typed with $[l:[]]$ :

$$
\frac{\frac{\emptyset[x \leftarrow[l:[]]] \vdash x:[l:[]] \quad[l:[]] \leq[]}{\emptyset[x \leftarrow[l:[]]] \vdash x:[]}}{\emptyset \vdash o^{\prime}:[l:[]]}
$$

Consider then the object $o^{\prime \prime} \equiv[l=\varsigma(y) b]$, where $b \equiv y . l \Leftarrow \varsigma(x) x$. The expression $o^{\prime \prime} . l$ can be typed as follows, with both $x$ and $y$ implicitly typed with $[l:[]]$ :

$$
\frac{\frac{\emptyset[y \leftarrow[l:[]]] \vdash y:[l:[]] \quad \emptyset[y \leftarrow[l:[]], x \leftarrow[l:[]]] \vdash x:[]}{\emptyset[y \leftarrow[l:[]]] \vdash b:[l:[]][l:[]] \leq[]]}}{\emptyset[y \leftarrow[l:[]]] \vdash b:[]} \frac{\emptyset \vdash o^{\prime \prime}:[l:[]]}{\emptyset \vdash o^{\prime \prime} . l:[] .}
$$

Consider finally the object [ ]. Trying to type the expression [ ].l will fail because rule (2) can only give [] the type [ ], so rule (3) cannot be applied afterwards. 


\section{From Rules to Constraints}

In this section we prove that the type inference problem is log space equivalent to solving a finite system of type constraints. The constraints isolate the essential combinatorial structure of the type inference problem.

Definition 4.1 Given a denumerable set of variables, an $A C$-system (Abadi/Cardelli-system) is a finite set of inequalities $W \leq W^{\prime}$, where $W$ and $W^{\prime}$ are of the forms $V$ or $\left[l_{i}: V_{i}{ }^{i \in 1 . . n}\right]$, and where $V, V_{1}, \ldots, V_{n}$ are variables. If $L$ maps variables to types, then define $\widetilde{L}$ as follows:

$$
\widetilde{L}(W)= \begin{cases}{\left[l_{1}: L\left(V_{1}\right), \ldots, l_{n}: L\left(V_{n}\right)\right]} & \text { if } W \text { is of the form }\left[l_{i}: V_{i} \quad i \in 1 . . n\right] \\ L(V) & \text { if } W \text { is a variable } V\end{cases}
$$

A solution for an AC-system is a map $L$ from variables to types such that for all $W \leq W^{\prime}$ in the AC-system, $\widetilde{L}(W) \leq \widetilde{L}\left(W^{\prime}\right)$.

For examples of AC-systems, see Section 9.

We first prove that the type inference problem is log space reducible to solving AC-systems.

Given an untyped $\varsigma$-term $c$, assume that it has been $\alpha$-converted so that all bound variables are distinct. We will now generate an $\mathrm{AC}$-system from $c$ where the bound variables of $c$ are a subset of the variables in the constraint system. This will be convenient in the statement and proof of Lemma 4.2 below. Let $X$ be the set of bound variables in $c$; let $Y$ be a set of variables disjoint from $X$ consisting of one variable $\llbracket b \rrbracket$ for each occurrence of a subterm $b$ of $c$; and let $Z$ be a set of variables disjoint from $X$ and $Y$ constisting of one variable $\left\langle a . l_{j}\right\rangle$ for each occurrence of a subterm $a . l_{j}$ of $c$. (The notations $\llbracket b \rrbracket$ and $\left\langle a . l_{j}\right\rangle$ are ambiguous because there may be more than one occurrence of the term $b$ or $a . l_{j}$ in $c$. However, it will always be clear from context which occurrence is meant.) Notice that there are two variables $\left\langle a . l_{j}\right\rangle$ and $\llbracket a . l_{j} \rrbracket$ for each occurrences of a subterm $a . l_{j}$ of $c$. Intuitively, $\left\langle a . l_{j}\right\rangle$ denotes the type of $a . l_{j}$ before subtyping, and $\llbracket a . l_{j} \rrbracket$ denotes the type of $a . l_{j}$ after subtyping. We generate the following AC-system of inequalities over $X \cup Y \cup Z$ :

- for every occurrence in $c$ of a bound variable $x$, the inequality

$$
x \leq \llbracket x \rrbracket
$$


- for every occurrence in $c$ of a subterm of the form $\left[l_{i}=\varsigma\left(x_{i}\right) b_{i}{ }^{i \in 1 . . n}\right]$, the inequality

$$
\left[l_{i}: \llbracket b_{i} \rrbracket{ }^{i \in 1 . . n}\right] \leq \llbracket\left[l_{i}=\varsigma\left(x_{i}\right) b_{i}{ }^{i \in 1 . . n}\right] \rrbracket
$$

and for every $i \in 1$..n, the equalities

$$
\left.x_{i}=\left[l_{i}: \llbracket b_{i}\right]^{i \in 1 . . n}\right]
$$

- for every occurrence in $c$ of a subterm of the form $a . l_{j}$, the inequalities

$$
\begin{gathered}
\llbracket a \rrbracket \leq\left[l_{j}:\left\langle a \cdot l_{j}\right\rangle\right] \\
\left\langle a . l_{j}\right\rangle \leq \llbracket a . l_{j} \rrbracket
\end{gathered}
$$

- for every occurrence in $c$ of a subterm of the form $a . l_{j} \Leftarrow \varsigma(x) b$, the constraints

$$
\begin{gathered}
\llbracket a \rrbracket \leq \llbracket a \cdot l_{j} \Leftarrow \varsigma(x) b \rrbracket \\
\llbracket a \rrbracket=x \\
\llbracket a \rrbracket \leq\left[l_{j}: \llbracket b \rrbracket\right]
\end{gathered}
$$

In (8) to (15), each equality $A=B$ denotes the two inequalities $A \leq B$ and $B \leq A$.

Denote by $C(c)$ the AC-system of constraints generated from $c$ in this fashion. For a s-term $c$ of size $n$, the $\mathrm{AC}$-system $C(c)$ is of size $O(n)$, and it is generated using $O(\log n)$ space. We show below that the solutions of $C(c)$ over $T$ correspond to the possible type annotations of $c$ in a sense made precise by Lemma 4.2. For examples of AC-systems generated from $\varsigma$-terms, see Section 9.

The constraints are motivated by the forms of the corresponding type rules. The reason for the use of $\leq$ in four of the constraint rules can be summarized as follows:

- Given a type derivation, we can find a particular type derivation where the subsumption rule (5) is used exactly once for each occurrence of a subterm. 
This explains the use of $\leq$ in the constraints (8), (9), (12), (13). For example, consider an occurrence in $c$ of a variable $x$. If the constraint $x \leq \llbracket x \rrbracket$ has solution $L$, then we can construct the type derivation

$$
\frac{L \vdash x: L(x) \quad L(x) \leq L(\llbracket x \rrbracket)}{L \vdash x: L(\llbracket x \rrbracket) .}
$$

The use of $\leq$ in the constraints (11) and (15) is motivated by the rules (6) and $(7)$.

Notice that we cannot replace the constraints (11) and (12) by the single constraint

$$
\llbracket a \rrbracket \leq\left[l_{j}: \llbracket a \cdot l_{j} \rrbracket\right]
$$

To see this, let $a . l_{j}$ occur in $c$ and suppose $C\left(a . l_{j}\right)$ has solution $L$ (so in particular, (11) and (12) has solution $L$ ). Consider the type derivation

$$
\frac{\frac{L \vdash a: L(\llbracket a \rrbracket) \quad L(\llbracket a \rrbracket) \leq\left[l_{j}: L\left(\left\langle a . l_{j}\right\rangle\right)\right]}{L \vdash a:\left[l_{j}: L\left(\left\langle a . l_{j}\right\rangle\right)\right]}}{\frac{L \vdash a . l_{j}: L\left(\left\langle a . l_{j}\right\rangle\right) \quad L\left(\left\langle a \cdot l_{j}\right\rangle\right) \leq L\left(\llbracket a \cdot l_{j} \rrbracket\right)}{L \vdash a . l_{j}: L\left(\llbracket a . l_{j} \rrbracket\right)}}
$$

Clearly, $L(\llbracket a \rrbracket) \downarrow l_{j}$ need not be equal to $L\left(\llbracket a . l_{j} \rrbracket\right)$. With the constraint (16), however, they are forced to be equal.

Let $E$ be a type environment assigning a type to each variable occurring freely in $c$. If $L$ is a function assigning a type to each variable in $X \cup Y \cup Z$, we say that $L$ extends $E$ if $E$ and $L$ agree on the domain of $E$.

If $b$ is an annotated $\varsigma$-term, then we let $\bar{b}$ denote the corresponding untyped term. Moreover, we let $\widehat{b}$ be the partial function that maps each bound variable in $b$ to its type annotation.

Lemma 4.2 The judgement $E \vdash c: A$ is derivable if and only if there exists a solution $L$ of $C(\bar{c})$ extending both $E$ and $\widehat{c}$, such that $L(\llbracket \bar{c} \rrbracket)=A$. In particular, if $c$ is closed, then $c$ is well-typed with type $A$ if and only if there exists a solution $L$ of $C(\bar{c})$ extending $\widehat{c}$ such that $L(\llbracket \bar{c} \rrbracket)=A$.

Proof. We first prove that if $C(\bar{c})$ has a solution $L$ extending both $E$ and $\widehat{c}$, then $L \vdash c: L(\llbracket \bar{c} \rrbracket)$ is derivable. We proceed by induction on the structure of $c$. 
For the base case, $L \vdash x: L(\llbracket x \rrbracket)$ is derivable using rules (1) and (5), since $L(x) \leq L(\llbracket x \rrbracket)$.

For the induction step, consider first $\left[l_{i}=\varsigma\left(x_{i}\right){\overline{b_{i}}}^{i \in 1 . . n}\right]$. Let $A=$ $\left[l_{i}: L\left(\llbracket \overline{b_{i}} \rrbracket\right)^{i \in 1 . . n}\right]$. To derive $L \vdash\left[l_{i}=\varsigma\left(x_{i}: L\left(x_{i}\right)\right) b_{i}{ }^{i \in 1 . . n}\right]: L\left(\llbracket\left[l_{i}=\right.\right.$ $\left.\left.\varsigma\left(x_{i}\right) \bar{b}_{i}{ }^{i \in 1 . . n}\right] \rrbracket\right)$, by rule (5) and the fact that $A \leq L\left(\llbracket\left[l_{i}=\varsigma\left(x_{i}\right) \overline{b_{i}} \quad{ }^{i \in 1 . . n}\right] \rrbracket\right)$, it suffices to derive $L \vdash\left[l_{i}=\varsigma\left(x_{i}: L\left(x_{i}\right)\right) b_{i}{ }^{i \in 1 . . n}\right]: A$. From the fact that $L\left(x_{i}\right)=A$ for every $i \in 1 . . n$, it suffices to derive $L \vdash\left[l_{i}=\varsigma\left(x_{i}: A\right) b_{i}{ }^{i \in 1 . . n}\right]$ : $A$. The side condition of rule (2) is clearly satisfied, so it suffices to derive, for each $i \in 1 . . n, L\left[x_{i} \leftarrow A\right] \vdash b_{i}: L\left(\llbracket \overline{b_{i}} \rrbracket\right)$, or in other words, $L \vdash b_{i}: L\left(\llbracket \overline{b_{i}} \rrbracket\right)$. But since $L$ is a solution of $C\left(\left[l_{i}=\varsigma\left(x_{i}\right) \overline{b_{i}} \quad i \in 1 . . n\right]\right)$, it is also a solution of $C\left(\overline{b_{i}}\right)$ for each $i \in 1$..n, thus the desired derivations are provided by the induction hypothesis.

Now consider $a . l_{j}$. Since $L$ is a solution of $C\left(\bar{a} . l_{j}\right)$, it is also a solution of $C(\bar{a})$. From the induction hypothesis, we obtain a derivation of $L \vdash a$ : $L(\llbracket \bar{a} \rrbracket)$. By rule $(3)$ and the fact that $L(\llbracket \bar{a} \rrbracket) \leq\left[l_{j}: L\left(\left\langle\bar{a} \cdot l_{j}\right\rangle\right)\right]$, we obtain a derivation of $L \vdash a . l_{j}: L\left(\left\langle\bar{a} . l_{j}\right\rangle\right)$. Using rule $(5)$ and the fact that $L\left(\left\langle\bar{a} . l_{j}\right\rangle\right) \leq$ $L\left(\llbracket \bar{a} . l_{j} \rrbracket\right)$, we then obtain a derivation of $L \vdash a . l_{j}: L\left(\llbracket \bar{a} \cdot l_{j} \rrbracket\right)$.

Finally consider $a . l_{j} \Leftarrow \varsigma(x: L(x)) b$. Let $A=L(\llbracket \bar{a} \rrbracket)$. To derive $L \vdash$ $a . l_{j} \Leftarrow \varsigma(x: L(x)) b: L\left(\llbracket \bar{a} . l_{j} \Leftarrow \varsigma(x) \bar{b} \rrbracket\right)$, by rule $(5)$ and the fact that $A \leq$ $L\left(\llbracket \bar{a} . l_{j} \Leftarrow \varsigma(x) \bar{b} \rrbracket\right)$, it suffices to derive $L \vdash a . l_{j} \Leftarrow \varsigma(x: L(x)) b: A$. From the fact that $A=L(x)$, it suffices to derive $L \vdash a . l_{j} \Leftarrow \varsigma(x: A) b: A$. The side condition of rule $(7)$ is satisfied because $A \leq\left[l_{j}: L(\llbracket \bar{b} \rrbracket)\right]$, so it suffices to derive $L \vdash a: A$ and $L[x \leftarrow A] \vdash b: L(\llbracket \bar{b} \rrbracket)$, or in other words $L \vdash a: L(\llbracket \bar{a} \rrbracket)$ and $L \vdash b: L(\llbracket \bar{b} \rrbracket)$. But since $L$ is a solution of $C\left(\bar{a} \cdot l_{j} \Leftarrow \varsigma(x) \bar{b}\right)$, it is also a solution of $C(\bar{a})$ and $C(\bar{b})$, thus the desired derivations are provided by the induction hypothesis.

We then prove that if $E \vdash c: A$ is derivable, then there exists a solution $L$ of $C(\bar{c})$ extending both $E$ and $\widehat{c}$.

Suppose $E \vdash c: A$ is derivable, and consider a derivation of minimal length. Since the derivation is minimal, there is exactly one application of the rule (1) involving a particular occurrence of a variable $x$, exactly one application of the rule (2) involving a particular occurrence of a subterm $\left[l_{i}=\varsigma\left(x_{i}: A\right) b_{i}{ }^{i \in 1 . . n}\right]$, exactly one application of the rule (3) involving a particular occurrence of a subterm $a . l_{j}$, and exactly one application of the rule (4) involving a particular occurrence of a subterm $a . l_{j} \Leftarrow \varsigma(x: A) b$. In the case of a variable $x$, there is a unique type $B$ such that $F(x)=B$ for 
any $F$ such that a judgement $F \vdash a: B^{\prime}$ appears in the derivation for some occurrence of a subterm $a$ of $\varsigma(x: A) b$; this can be proved by induction on the structure of the derivation of $F \vdash a: B^{\prime}$. Finally, there can be at most one application of the rule (5) involving a particular occurrence of any subterm; if there were more than one, they could be combined using the transitivity of $\leq$ to give a shorter derivation.

Now construct $L$ as follows. For every free variable $x$ of $c$ define $L(x)=$ $E(x)$. For every bound variable $x$, let $\varsigma(x: A) b$ be the method in which it is bound, and define $L(x)=A$. For every occurrence of a subterm $a$ of $c$, find the last judgement in the derivation of the form $F \vdash a: B$ involving that occurrence of $a$, and define $L(\llbracket \bar{a} \rrbracket)=B$. Intuitively, the last judgement of the form $F \vdash a: B$ means the judgement after the use of subsumption. Finally, for every occurrence of a subterm $a . l_{j}$ of $c$, find the unique application of the rule (3) deriving $F \vdash a . l_{j}: B_{j}$, and define $L\left(\left\langle a . l_{j}\right\rangle\right)=B_{j}$.

Certainly $L$ extends $E$ and $\widehat{c}$ and $L(\llbracket \bar{c} \rrbracket)=A$. We now show that $L$ is a solution of $C(\bar{c})$.

For an occurrence of a bound variable $x$, there are two cases. Suppose first that the variable is bound in a method that occurs in an object declaration. Find the unique application of the rule (2) deriving the judgement $F \vdash$ $\left[l_{i}=\varsigma\left(x_{i}: A\right) b_{i}{ }^{i \in 1 . . n}\right]: A$ from a family of premises where one of them is $F[x \leftarrow A] \vdash b: B_{i}$. Then $L(x)=A$. The rule (1) must have been applied to obtain a judgement of the form $G \vdash x: L(x)$ and only rule (5) applied to that occurrence of $x$ thereafter, thus $L(x) \leq L(\llbracket x \rrbracket)$. Suppose then that the variable is bound in a method that occurs in a method override. Find the unique application of the rule (4) deriving the judgement $F \vdash a . l_{j} \Leftarrow \varsigma(x$ : $A) b: A$ from two premises where one of them is $F[x \leftarrow A] \vdash b: B_{j}$. As before, we get that $L(x) \leq L(\llbracket x \rrbracket)$.

For an occurrence of a subterm of the form $\left[l_{i}=\varsigma\left(x_{i}: A\right) b_{i}{ }^{i \in 1 . . n}\right]$, find the unique application of the rule (2) deriving the judgement $F \vdash\left[l_{i}=\varsigma\left(x_{i}\right.\right.$ : $\left.A) b_{i}{ }^{i \in 1 . . n}\right]: A$ from the premises $F\left[x_{i} \leftarrow A\right] \vdash b_{i}: B_{i}$, where $A=\left[l_{i}\right.$ : $\left.L\left(\llbracket \overline{b_{i}} \rrbracket\right){ }^{i \in 1 . . n}\right]$. Then $L\left(x_{i}\right)=A$, and $A \leq L\left(\llbracket\left[l_{i}=\varsigma\left(x_{i}\right) \bar{b}_{i} \quad i \in 1 . . n\right] \rrbracket\right)$.

For an occurrence of a subterm of the form $a . l_{j}$, find the unique application of the rule (3) deriving the judgement $F \vdash a . l_{j}: B_{j}$ from the premise $F \vdash a:\left[l_{i}: B_{i}{ }^{i \in 1 . . n}\right]$. Then $L(\llbracket \bar{a} \rrbracket)=\left[l_{i}: B_{i}{ }^{i \in 1 . . n}\right]$ and $B_{j}=L\left(\left\langle\bar{a} \cdot l_{j}\right\rangle\right) \leq$ $L\left(\llbracket \bar{a} . l_{j} \rrbracket\right)$. Thus, $L(\llbracket \bar{a} \rrbracket) \leq\left[l_{j}: L\left(\left\langle\bar{a} \cdot l_{j}\right\rangle\right)\right]$, by the definition of $\leq$. 
Finally for an occurrence of a subterm of the form $a . l_{j} \Leftarrow \varsigma(x: A) b$, find the unique application of the rule (4) deriving the judgement $F \vdash a . l_{j} \Leftarrow$ $\varsigma(x: A) b: A$ from the premises $F \vdash a: A$ and $F[x \leftarrow A] \vdash b: B_{j}$, where $A=\left[l_{i}: B_{i}{ }^{i \in 1 . . n}\right]$. Then $L(\llbracket \bar{a} \rrbracket)=A \leq L\left(\llbracket \bar{a} . l_{j} \Leftarrow \varsigma(x) \bar{b} \rrbracket\right)$, and $A=L(x)$. Moreover, $L(\llbracket \bar{b} \rrbracket)=B_{j}$, so $L(\llbracket \bar{a} \rrbracket) \leq\left[l_{j}: L(\llbracket \bar{b} \rrbracket)\right]$, by the definition of $\leq$.

We then prove that solving AC-systems is log space reducible to the type inference problem.

Let $C$ be an AC-system. Suppose $L$ assigns a type to each variable in $C$. Recall that for an inequality $W \leq W^{\prime}$ in $C$, both $W$ and $W^{\prime}$ are of the forms $V$ or $\left[l_{i}: V_{i}{ }^{i \in 1 . . n}\right]$, where $V, V_{1}, \ldots, V_{n}$ are variables. Define

$$
\begin{aligned}
& A_{L}^{C}=\left[l_{V}: L(V) \text { for each variable } V \text { in } C\right. \\
& l_{R}: A_{R} \quad \text { for each } R \text { in } C \text { of the form }\left[l_{i}: V_{i} \quad \text { i } \quad \text {...n }\right] \\
& \text { and where } A_{R}=\left[l_{i}: L\left(V_{i}\right) \text { ie1..n }\right] \\
& l_{I}: \widetilde{L}\left(W^{\prime}\right) \text { for each inequality } I \text { in } C \text { of the form } W \leq W^{\prime} \\
& \text { ] } \\
& \text { Define also } \\
& c_{L}^{C}=\left[l_{V}=\varsigma\left(x_{V}: A_{L}^{C}\right)\left(x_{V} \cdot l_{V}\right)\right. \\
& \text { for each variable } V \text { in } C \\
& l_{R}=\varsigma\left(x_{R}: A_{L}^{C}\right)\left[l_{i}=\varsigma\left(x_{i}: A_{R}\right)\left(x_{R} \cdot l_{V_{i}}\right) \quad i \in 1 . . n\right] \\
& \text { for each } R \text { in } C \text { of the form }\left[l_{i}: V_{i} \quad \text { i } \quad \text {...n }\right] \\
& \text { and where } A_{R}=\left[l_{i}: L\left(V_{i}\right) \text { ie1..n }\right] \\
& l_{I}=\varsigma\left(x_{I}: A_{L}^{C}\right)\left(\left(x_{I} \cdot l_{W^{\prime}} \Leftarrow \varsigma\left(y: A_{L}^{C}\right)\left(x_{I} \cdot l_{W}\right)\right) \cdot l_{W^{\prime}}\right) \\
& \text { for each inequality } I \text { in } C \text { of the form } W \leq W^{\prime}
\end{aligned}
$$

Notice that $A_{L}^{C}$ and $c_{L}^{C}$ can be generated in $\log$ space.

Lemma 4.3 $C$ has solution $L$ if and only if $\emptyset \vdash c_{L}^{C}: A_{L}^{C}$ is derivable.

Proof. We first prove that if $C$ has solution $L$ then $\emptyset \vdash c_{L}^{C}: A_{L}^{C}$ is derivable. Using rule (2), we get that we need to derive judgements of the form $E\left[x_{i} \leftarrow A_{L}^{C}\right] \vdash b_{i}: B_{i}$, where each $b_{i}$ is the body of a method in $c_{L}^{C}$ and $B_{i}$ is the type in the corresponding entry of $A_{L}^{C}$.

Consider first an entry in $c_{L}^{C}$ corresponding to a variable $V$ in $C$. The method body is $x_{V} \cdot l_{V}$ and it is clearly derivable that $E\left[x_{V} \leftarrow A_{L}^{C}\right] \vdash x_{V} \cdot l_{V}$ : $L(V)$.

Consider then an entry in $c_{L}^{C}$ corresponding to $R$ in $C$ of the form $\left[l_{i}\right.$ : $\left.V_{i}{ }^{i \in 1 . . n}\right]$. The method body is $\left[l_{i}=\varsigma\left(x_{i}: A_{R}\right)\left(x_{R} \cdot l_{V_{i}}\right)^{i \in 1 . . n}\right]$ and it is 
clearly derivable that $E\left[x_{R} \leftarrow A_{L}^{C}\right] \vdash\left[l_{i}=\varsigma\left(x_{i}: A_{R}\right)\left(x_{R} \cdot l_{V_{i}}\right)^{i \in 1 . . n}\right]:\left[l_{i}:\right.$ $\left.L\left(V_{i}\right)^{i \in 1 . . n}\right]$.

Consider finally an entry in $c_{L}^{C}$ corresponding to an inequality $I$ of the form $W \leq W^{\prime}$. Using that $\widetilde{L}(W) \leq \widetilde{L}\left(W^{\prime}\right)$, it is straightforward to derive $E\left[x_{I} \leftarrow A_{L}^{C}\right] \vdash\left(\left(x_{I} \cdot l_{W^{\prime}} \Leftarrow \varsigma\left(y: A_{L}^{C}\right)\left(x_{I} \cdot l_{W}\right)\right) . l_{W^{\prime}}\right): L\left(W^{\prime}\right)$.

We then prove that if $\emptyset \vdash c_{L}^{C}: A_{L}^{C}$ is derivable then $C$ has solution $L$. Consider any inequality $I$ in $C$ of the form $W \leq W^{\prime}$. Let $\left(x_{I} . l_{W^{\prime}} \Leftarrow \varsigma(y\right.$ : $\left.\left.A_{L}^{C}\right)\left(x_{I} . l_{W}\right)\right) \cdot l_{W}$, be the body of the corresponding method in $c_{L}^{C}$. Clearly, any derivation involving this $\varsigma$-term must contain the judgement $E \vdash x_{I} . l_{W}$ : $\widetilde{L}\left(W^{\prime}\right)$, where $E$ maps both of the variables $x_{I}$ and $y$ to $A_{L}^{C}$. Consider a derivation where rule (5) is used exactly once for each occurrence of a subterm. The judgement $E \vdash x_{I}, l_{W}: \widetilde{L}\left(W^{\prime}\right)$ has been derived using rule (5), so the hypothesis must have had the form $E \vdash x_{I} \cdot l_{W}: B$, where $B \leq \widetilde{L}\left(W^{\prime}\right)$. That judgement must have been derived using rule (3), so the hypothesis must have had the form $E \vdash x_{I}: R$, where $R \leq\left[l_{W}: B\right]$. That judgement has been derived using rule (5), so the hypothesis must have had the form $E \vdash x_{I}: A_{L}^{C}$, where $A_{L}^{C} \leq R$. By combining $A_{L}^{C} \leq R$ and $R \leq\left[l_{W}: B\right]$, we get that $B=A_{L}^{C} \downarrow l_{W}$. Notice that $A_{L}^{C} \downarrow l_{W}=\widetilde{L}(W)$, so $\widetilde{\widetilde{L}}(W)=B$. Combining that with $B \leq \widetilde{L}\left(W^{\prime}\right)$ gives $\widetilde{L}(W) \leq \widetilde{L}\left(W^{\prime}\right)$.

For example, let $C$ be the AC-system consisting of the single constraint

$$
V \leq[l: W]
$$

Moreover, let $L$ be such that

$$
\begin{aligned}
L(V) & =[l: B] \\
L(W) & =B
\end{aligned}
$$

for some type $B$. We then get:

$$
\begin{aligned}
A_{L}^{C}= & {\left[l_{V}:[l: B]\right.} \\
& l_{W}: B \\
& l_{[l: W]}:[l: B] \\
& l_{V \leq[l: W]}:[l: B] \\
& \begin{array}{l} 
\\
c_{L}^{C}=
\end{array} l_{V}=\varsigma\left(x_{V}: A_{L}^{C}\right)\left(x_{V} \cdot l_{V}\right)
\end{aligned}
$$




$$
\begin{aligned}
& l_{W}=\varsigma\left(x_{W}: A_{L}^{C}\right)\left(x_{W} \cdot l_{W}\right) \\
& l_{[l: W]}=\varsigma\left(x_{[l: W]}: A_{L}^{C}\right)\left[l=\varsigma(x:[l: B])\left(x_{[l: W]} \cdot l_{W}\right)\right] \\
& l_{V \leq[l: W]}=\varsigma\left(x_{V \leq[l: W]}: A_{L}^{C}\right) b \\
& \text { where } \\
& b=\left(x_{V \leq[l: W]} \cdot l_{[l: W]} \Leftarrow \varsigma\left(y: A_{L}^{C}\right)\left(x_{V \leq[l: W]} \cdot l_{V}\right)\right) \cdot l_{[l: W]}
\end{aligned}
$$

By combining Lemmas 4.2 and 4.3 we get the following result.

Theorem 4.4 The type inference problem is log space equivalent to solving AC-systems.

\section{From Constraints to Graphs}

In this section we prove that solving AC-systems is log space equivalent to solving a certain kind of constraint graphs. The graphs yield a convenient setting for applying algorithms like transitive closure.

Definition 5.1 A $A C$-graph is a directed graph $G=(N, S, L, \leq)$ consisting of two disjoint sets of nodes $N$ and $S$, and two disjoint sets of directed edges $L$ and $\leq$. Each edge in $L$ is labeled with some $l \in \mathcal{N}$, and each edge in $\leq$ is labeled with $\leq$. An AC-graph satisfies the properties:

- any $N$ node has finitely many outgoing $L$ edges, all to $S$ nodes, and those edges have distinct labels;

- any $S$ node has no outgoing $L$ edges;

For each $h: S \rightarrow T$, define $\widetilde{h}:(N \cup S) \rightarrow T$ as

$\widetilde{h}(u)= \begin{cases}{\left[l_{1}: h\left(v_{1}\right), \ldots, l_{n}: h\left(v_{n}\right)\right]} & \text { if } u \stackrel{l_{i}}{\rightarrow} v_{i} \text { are the } L \text { edges from } u \in N \\ h(u) & \text { if } u \in S\end{cases}$

A solution for $G$ is any map $h: S \rightarrow T$ such that if $u \stackrel{\leq}{\rightarrow}$, then $\widetilde{h}(u) \leq \widetilde{h}(v)$. The solution $h$ is finite if $h(s)$ is a finite set for all $s$. 
For examples of AC-graphs, see Section 9.

Recall that we represent a type as a set of strings. Note that a solvable AC-graph has a pointwise $\subseteq$-least solution. To see this, observe that the intersection of any non-empty family of solutions is itself a solution. Thus, provided that solutions exist, the intersection of all solutions is the pointwise $\subseteq$-least solution.

Theorem 5.2 Solving AC-systems is log space equivalent to solving $A C$ graphs.

Proof. Given an AC-system, we construct an AC-graph as follows. Associate a unique $N$ node with every subexpression of the form $\left[l_{i}: V_{i}{ }^{i \in 1 . . n}\right]$, and associate a unique $S$ node with every variable. From the node for $\left[l_{i}: V_{i}{ }^{i \in 1 . . n}\right]$, define for each $i \in 1 . . n$ an $L$ edge labeled $l_{i}$ to the node for $V_{i}$. Finally, define $\leq$ edges for the inequalities. Notice that the AC-graph can be generated in log space. Clearly, the resulting $\mathrm{AC}$-graph is solvable if and only if the AC-system is solvable.

Conversely, given an AC-graph, we construct an AC-system as follows. For every $\leq$ edge, generate the obvious inequality. Notice that the AC-system can be generated in log space. Clearly, the resulting AC-system is solvable if and only if the AC-graph is solvable.

For examples of AC-graphs generated from AC-systems, see Section 9.

The following two definitions provide the basis for the algorithms for solving AC-graphs that will be presented in Section 7. First we define the closure of an AC-graph, which makes the solutions (or lack thereof) explicit. Then we define a condition for solvability: well-formedness of the graph. The concepts of closure and well-formedness are brought together in Theorem 6.5 which says that a closed graph is solvable if and only if it is well-formed.

Definition 5.3 An AC-graph is closed if the edge relation $\leq$ is reflexive, transitive, and closed under the following rule which say that the dashed edge exists whenever the solid ones do:

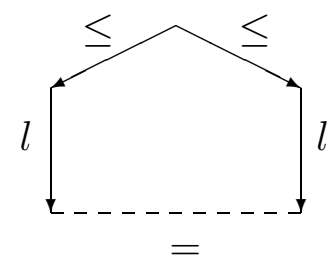


The $=$ edge denotes two $\leq$ edges, one in each direction.

The closure of an AC-graph $G$ is the smallest closed AC-graph containing $G$ as a subgraph.

Note that an AC-graph and its closure have the same set of solutions. To see this, observe that any solution of the closure of $G$ is also a solution of $G$, since $G$ has fewer constraints. Conversely, observe that the closure of $G$ can be constructed from $G$ by iterating the closure rules, and it follows inductively by the definition of $\leq$ that any solution of $G$ satisfies the additional constraints added by this process.

Definition 5.4 An AC-graph is well-formed if for any nodes $u, v \in N$ with $u \stackrel{s}{\leftrightarrows}$, if $v$ has an outgoing edge labeled $l$, then so does $u$.

\section{From Graphs to Automata}

In this section we define an automaton $\mathcal{M}$ and prove our main result. This automaton will be used to characterize the canonical assignment of types to nodes of a given AC-graph.

Definition 6.1 Let an AC-graph $G=(N, S, L, \leq)$ be given. The automaton $\mathcal{M}$ is defined as follows. The input alphabet of $\mathcal{M}$ is $\mathcal{N}$ (the set of method names). The states of $\mathcal{M}$ are $N \cup S$. We use $p, q, s, u, v, w$ to range over states. The transitions are defined as follows.

$$
\begin{aligned}
& v \stackrel{\epsilon}{\rightarrow} v^{\prime} \text { if } v \stackrel{\leftrightarrows}{\rightarrow} v^{\prime} \text { in } G \\
& v \stackrel{l}{\rightarrow} v^{\prime} \text { if } v \stackrel{l}{\rightarrow} v^{\prime} \text { in } G
\end{aligned}
$$

If $p$ and $q$ are states of $\mathcal{M}$ and $\alpha \in \mathcal{N}^{*}$, we write $p \stackrel{\alpha}{\rightarrow} q$ if the automaton can move from state $p$ to state $q$ under input $\alpha$, including possible $\epsilon$-transitions.

The automaton $\mathcal{M}_{s}$ is the automaton $\mathcal{M}$ with start state $s$. All states are accept states; thus the language accepted by $\mathcal{M}_{s}$ is the set of strings $\alpha$ for which there exists a state $p$ such that $s \stackrel{\alpha}{\rightarrow} p$. We denote this language by $\mathcal{L}(s)$.

The set $\mathcal{L}(s)$ is clearly nonempty, since $s \stackrel{\epsilon}{\rightarrow} s$ is in $\mathcal{M}$, and it is prefixclosed since all states in $\mathcal{M}$ are accept states. Moreover, $\mathcal{L}(s)$ is regular: each of the finitely many states in $\mathcal{M}$ corresponds to a subtree in $\mathcal{L}(s)$. Hence, $\mathcal{L}(s) \in T$. 
The intuition motivating the definition of $\mathcal{M}$ is that we want to identify the conditions that require a path to exist in any solution. Thus $\mathcal{L}(s)$ is the set of $\alpha$ that must be there; this intuition is made manifest in the following lemma.

Recall from Section 3 that $A \downarrow \alpha$ is the subtree of $A$ at $\alpha$ if $\alpha \in A, \emptyset$ if not.

Lemma 6.2 Let $G$ be an $A C$-graph. If $h: S \rightarrow T$ is any solution and $s \stackrel{\alpha}{\rightarrow} p$ in $\mathcal{M}$, then $\alpha \in \widetilde{h}(s)$ and $\widetilde{h}(s) \downarrow \alpha \leq \widetilde{h}(p)$.

Proof. We proceed by induction on the number of transitions. If this is zero, then $p=s$ and $\alpha=\epsilon$, and the result is immediate. Otherwise, assume that $s \stackrel{\alpha}{\rightarrow} p$ in $\mathcal{M}$ and the lemma holds for this sequence of transitions. We argue by cases, depending on the form of the next transition out of $p$.

Consider first a transition $p \stackrel{\epsilon}{\rightarrow} q$. Then $p \stackrel{\leq}{\rightarrow} q$ in $G$, so $\widetilde{h}(p) \leq \widetilde{h}(q)$. By the induction hypothesis, we get $\alpha \epsilon=\alpha \in \widetilde{h}(s)$ and

$$
\widetilde{h}(s) \downarrow \alpha \epsilon=\widetilde{h}(s) \downarrow \alpha \leq \widetilde{h}(p) \leq \widetilde{h}(q) .
$$

Consider then a transition $p \stackrel{l}{\rightarrow} q$. Then $p \stackrel{l}{\rightarrow} q$ in $G$, so $\widetilde{h}(p) \downarrow l=\widetilde{h}(q)$. Thus, $l \in \widetilde{h}(p)$. By the induction hypothesis we get $\widetilde{h}(p) \subseteq \widetilde{h}(s) \downarrow \alpha$, so $l \in \widetilde{h}(s) \downarrow \alpha$, hence $\alpha l \in \widetilde{h}(s)$. We also get

$$
\widetilde{h}(s) \downarrow \alpha l=(\widetilde{h}(s) \downarrow \alpha) \downarrow l=\widetilde{h}(p) \downarrow l=\widetilde{h}(q)
$$

using the definition of $\leq$.

Lemma 6.3 Let $G$ be a closed AC-graph. Suppose that $G$ contains the edges $u \stackrel{\leq}{\rightarrow} \stackrel{l}{\rightarrow} w$. Then $\widetilde{\mathcal{L}}(u) \downarrow l=\widetilde{\mathcal{L}}(w)$.

Proof. Follows from $G$ being closed.

Lemma 6.4 Let $G$ be a closed $A C$-graph. Suppose that $G$ contains the edge $u_{1} \leqq u_{2}$. If $l \in \widetilde{\mathcal{L}}\left(u_{1}\right)$ and $l \in \widetilde{\mathcal{L}}\left(u_{2}\right)$, then $\widetilde{\mathcal{L}}\left(u_{1}\right) \downarrow l=\widetilde{\mathcal{L}}\left(u_{2}\right) \downarrow l$.

Proof. For each $i \in 1 . .2$ we observe that for any node $w_{i}$ and edges $u_{i} \stackrel{\leq}{\leftrightarrows} v_{i} \stackrel{l}{\rightarrow} w_{i}$, we get from Lemma 6.3 that $\widetilde{\mathcal{L}}\left(u_{i}\right) \downarrow l=\widetilde{\mathcal{L}}\left(w_{i}\right)$. Thus, it is sufficient to choose two such nodes $w_{1}$ and $w_{2}$, and prove that $\widetilde{\mathcal{L}}\left(w_{1}\right)=\tilde{\mathcal{L}}\left(w_{2}\right)$. This choice is possible since $l \in \widetilde{\mathcal{L}}\left(u_{1}\right)$ and $l \in \widetilde{\mathcal{L}}\left(u_{2}\right)$. From $G$ being closed we get that there are edges $w_{1} \leqq w_{2}$ and $w_{2} \leqq w_{1}$. Thus, $\widetilde{\mathcal{L}}\left(w_{1}\right)=\widetilde{\mathcal{L}}\left(w_{2}\right)$ indeed holds. 
Theorem 6.5 A closed AC-graph is solvable if and only if it is well-formed. If it is solvable, then $\mathcal{L}$ is the pointwise $\subseteq$-least solution.

Proof. Let $G$ be a closed AC-graph. Clearly, if $G$ is solvable, then it is well-formed.

Assume now that $G$ is well-formed. To show that $\mathcal{L}$ is a solution for $G$, we need to prove that if $u \stackrel{s}{\Rightarrow}$ in $G$, then $\widetilde{\mathcal{L}}(u) \leq \widetilde{\mathcal{L}}(v)$. Suppose $l \in \widetilde{\mathcal{L}}(v)$. We must prove that $l \in \widetilde{\mathcal{L}}(u)$ and $\widetilde{\mathcal{L}}(u) \downarrow l=\widetilde{\mathcal{L}}(v) \downarrow l$. If we can prove $l \in \widetilde{\mathcal{L}}(u)$, then $\widetilde{\mathcal{L}}(u) \downarrow l=\widetilde{\mathcal{L}}(v) \downarrow l$ follows from Lemma 6.4 .

First, if $u \in N$, then from $G$ being well-formed, we get $u \stackrel{l}{\rightarrow} w$ in $G$ for some $w$. Thus, $l \in \widetilde{\mathcal{L}}(u)$. Second, if $u \in S$, then from $u \leqq v$, we get from Lemma 6.3 that $l \in \widetilde{\mathcal{L}}(u)$.

To show that $\mathcal{L}$ is the pointwise $\subseteq$-least solution, we need to show that for any solution $h: S \rightarrow T, \mathcal{L}(s) \subseteq h(s)$ for all $s$. This follows directly from Lemma 6.2.

Intuitively, the pointwise $\subseteq$-least solution is the one where the types contain only those fields that are absolutely required by the type rules.

\section{Algorithms}

We first demonstrate how to close an AC-graph and how to check if an AC-graph is well-formed. Then we proceed to presenting the type inference algorithms.

\subsection{Closure}

To compute the closure of an AC-graph $(N, S, L, \leq)$, we use four data structures:

1. ITC: a data structure for incremental transitive closure. It maintains the transitive closure of a graph of $\leq$ edges during edge insertions. Specifically, it maintains an adjacency matrix $T C$ such that after each edge insertion, $T C[x, y]=1$ if and only if there is a path from $x$ to $y$ in the graph, and $T C[x, y]=0$ otherwise. An edge insertion may result in the addition of more edges, and so on, recursively. The insert 
operation returns a list of all edges that have been added, represented as node pairs. Initialization and maintenance of ITC can be done in $O\left(n^{3}\right)$ time, where $n$ is the number of nodes [15, 21].

2. PE: a data structure with potential $\leq$ edges. This data structure is computed in a preprocessing phase. It is a matrix of the same form as the adjacency matrix, but with each entry being a list of pairs of nodes. It is computed by, for each pair of $L$ edges $x_{1} \stackrel{l_{1}}{\rightarrow} y_{1}$ and $x_{2} \stackrel{l_{2}}{\rightarrow} y_{2}$, checking if $l_{1}=l_{2}$, and if so, appending $\left[\left(y_{1}, y_{2}\right),\left(y_{2}, y_{1}\right)\right]$ to $\mathbf{P E}\left[x_{1}, x_{2}\right]$. This takes $O\left(|L|^{2}\right)$ time. The idea is that, for $l_{1}=l_{2}$, if we later find edges $x \stackrel{\leq}{\rightarrow} x_{1}$ and $x \stackrel{\leq}{\rightrightarrows} x_{2}$ for some $x$, then we must insert $x_{1} \stackrel{l_{1}}{\rightarrow} y_{1}$ and $x_{2} \stackrel{l_{2}}{\rightarrow} y_{2}$ into ITC.

3. M: a matrix of the same form as the adjacency matrix. It is used to keep track of the accesses to PE. Specifically, $\mathbf{M}[x, y]=1$ if and only if we have accessed $\mathbf{P E}[x, y]$, and $\mathbf{M}[x, y]=0$ otherwise.

4. Q: the worklist. Specifically, Q is a list of pairs of nodes. Each pair $(x, y)$ indicates that $x \stackrel{\leftrightarrows}{\Rightarrow} y$ must be inserted into ITC.

The algorithm works as follows. First compute PE, initialize ITC to be the empty graph, initialize $\mathbf{M}$ to be the 0 -matrix, and initialize $\mathbf{Q}$ to be the list of $\leq$ edges of the input graph. Then repeat the following step until $\mathbf{Q}$ is empty:

- Let $(x, y)$ be a pair in $\mathbf{Q}$. Remove it and insert it into ITC. Let $R$ be the list which is returned by the insert operation. For each $(x, y)$ in $R$, find the immediate successors $z$ of $x$ in ITC, and if $\mathbf{M}[y, z]=0$, then set $\mathbf{M}[y, z]=1$ and set $\mathbf{M}[z, y]=1$, and append $\mathbf{P E}[y, z]$ to $\mathbf{Q}$.

Suppose $(N, S, L, \leq)$ is the input graph, and let $n$ be the number of nodes in the graph, that is, $n=|N|+|S|$. There can be $O\left(n^{2}\right)$ new edges in ITC, and for each one, we consider $O(n)$ immediate successors of a given node. For the graphs of our application, $|L| \in O(n)$, so for those the total running time of the algorithm is $O\left(n^{3}\right)$. 


\subsection{Well-formedness}

To check that an AC-graph is well-formed, do a depth-first search of the graph, maintaining for each node in $N$ both a set of labels of outgoing $L$ edges and a list of outgoing $\leq$ edges. Each time a node $u \in N$ is to be exited, check for each edge $u \stackrel{\leq}{\leftrightarrows} v$ that the label set for $v$ is a subset of the label set for $u$. The time spent is:

- The search itself takes time proportional to the number of edges, which is $O(|L|+|\leq|)$ time.

- The maintenance of label sets takes time proportional to inserting $|L|$ elements, which is $O(|L| \log |L|)$ time.

- The maintenance of lists of $\leq$ edges takes $O(|\leq|)$ time.

- The checks take $O\left(|L|^{2}\right)$ time. (There is either zero or one comparison of labels for each pair of $L$ edges.)

Suppose $(N, S, L, \leq)$ is the input graph, and let $n$ be the number of nodes in the graph, that is, $n=|N|+|S|$. For the graphs of our application, $|L| \in O(n)$, so for those the total running time of the algorithm is $O\left(n^{2}\right)$.

\subsection{Type inference}

We have shown that the type inference problem with recursive types and subtyping is log space equivalent to solving AC-graphs. Using the characterization of Theorem 6.5, we get a straightforward type inference algorithm:

Input: A s-term of size $n$.

1: Construct the corresponding AC-graph (in log space).

2: Close the graph (in $O\left(n^{3}\right)$ time).

3: Check if the resulting graph is well-formed (in $O\left(n^{2}\right)$ time).

4: If the graph is well-formed, then output "typable" together with the automaton $\mathcal{M}$ else output "not typable".

The entire algorithm requires $O\left(n^{3}\right)$ time. Every subterm of the input corresponds to a state $(s)$ in the automaton $\mathcal{M}$, and a suggestion for its type is represented by the language $\mathcal{L}(s)$. 
Consider then the type inference problem with finite types and subtyping. Clearly, this problem is log space equivalent to finding finite solutions to ACgraphs. By Theorem 6.5, there exists a finite solution if and only if $\mathcal{L}$ is a finite solution. Thus, we obtain a type inference algorithm by modifying step 4 of the above algorithm so that it checks that $\mathcal{L}$ is finite. To do that, we check for a cycle in $\mathcal{M}$ with at least one non- $\epsilon$ transition reachable from some $(s)$. This can be done in linear time in the size of the closed AC-graph using depth-first search. Thus, the entire algorithm requires $O\left(n^{3}\right)$ time.

Consider then the type inference problems without subsumption. This causes us to change the inequalities (8), (9), (12), and (13) to the corresponding equalities. Note that we leave the inequalities (11) and (15) unchanged. We can then repeat the development of Sections 4-6, but this time with a subclass of AC-systems, which we call SAC-systems (simple ACsystems). In SAC-systems, the allowed constraints are of the forms $W=W^{\prime}$ or $V \leq\left[l_{i}: V_{i}{ }^{i \in 1 . . n}\right]$, where $V, V_{1}, \ldots, V_{n}$ are variables, and $W, W^{\prime}$ are of the forms $V$ or $\left[l_{i}: V_{i}{ }^{i \in 1 . . n}\right]$. Similarly, there is a subclass of AC-graphs, which we call SAC-graphs. In SAC-graphs, most $\leq$ edges come in pairs, yielding "equality" edges. Specifically, consider an edge $u \leftrightarrows v$. Unless, $u$ is an $S$

node and $v$ is an $N$ node, then there is also an edge $v \stackrel{\leq}{\Rightarrow} u$. An SAC-graph is well-formed if it is well-formed as an AC-graph. Moreover, the closure of an SAC-graph $G$ is the smallest closed SAC-graph containing $G$ as a subgraph. Clearly, the "AC-closure" and the "SAC-closure" of an SAC-graph are the same. SAC-graphs can be solved using the above algorithms, in time $O\left(n^{3}\right)$, both with recursive and finite types.

In summary:

Theorem 7.1 All four type inference problems are solvable in $O\left(n^{3}\right)$ time.

\section{Completeness}

We now prove that all four type inference problems are P-hard under log space reductions. Clearly, it is sufficient to show that the two problems of solving SAC-systems with finite or recursive types are $\mathrm{P}$-hard. These two results are obtained by reductions of simple type inference for $\lambda$-calculus, and of the monotone circuit value problem, respectively, as explained in the following. 
We first consider finding finite solutions to SAC-systems. Simple type inference for $\lambda$-calculus is $\mathrm{P}$-complete under log space reductions [12]. It is $\log$ space equivalent to finding finite solutions to a finite set of equations of the forms $V=V^{\prime} \rightarrow V^{\prime \prime}$ or $V=C$, where $V, V^{\prime}, V^{\prime \prime}$ are variables, and $C$ is a constant. Variables range over the set of finite binary trees over the binary constructor $\rightarrow$ and some set of nullary constants. A solution is a map $L$ from variables to such trees such that all equations are satisfied. We can in $\log$ space transform such a set of equations into an equivalent SAC-system by translating $V=V^{\prime} \rightarrow V^{\prime \prime}$ into $V=\left[l: V^{\prime} \quad r: V^{\prime \prime}\right]$, and translating $V=C$ into $V=[]$. Thus, finding finite solutions to a $\mathrm{SAC}$-system is $\mathrm{P}$-hard.

We then consider finding arbitrary solutions to SAC-systems. A monotone circuit [7] is a directed acyclic graph $G$ whose nodes, called gates, are of five different kinds:

1. input gates with no in-edge and one out-edge;

2. and-gates with two in-edges and one out-edge;

3. or-gates with two in-edges and one out-edge;

4. fan-out gates with one in-edge and two out-edges; and

5. a single output gate with one in-edge and no out-edges.

Furthermore, all gates are reachable from the input gate, and the output gate is reachable from all gates.

Every assignment $a$ of truth values to the input gates of $G$ can be extended uniquely to a truth value assignment $\bar{a}$ to all gates of $G$ by defining:

1. if $n$ is an input gate, then $\bar{a}(n)=a(n)$;

2. if $n$ is an and-gate with predecessors $n^{\prime}$ and $n^{\prime \prime}$, then $\bar{a}(n)=\bar{a}\left(n^{\prime}\right) \wedge$ $\bar{a}\left(n^{\prime \prime}\right)$;

3. if $n$ is an or-gate with predecessors $n^{\prime}$ and $n^{\prime \prime}$, then $\bar{a}(n)=\bar{a}\left(n^{\prime}\right) \vee \bar{a}\left(n^{\prime \prime}\right)$;

4. if $n$ is a fan-out gate with predecessor $n^{\prime}$ and out-edges $n_{1}, n_{2}$, then $\bar{a}\left(n_{1}\right)=\bar{a}\left(n^{\prime}\right)$ and $\bar{a}\left(n_{2}\right)=\bar{a}\left(n^{\prime}\right) ;$ and

5. if $n$ is the output gate with predecessor $n^{\prime}$, then $\bar{a}(n)=\bar{a}\left(n^{\prime}\right)$. 
The monotone circuit value problem [16] is the problem of deciding, given a monotone circuit $G$ and an assignment $a$ to the input gates of $G$, whether $\bar{a}(n)=$ true for the output gate $n$. It is $\mathrm{P}$-complete under log-space reductions [16].

We will now give a log space reduction of the monotone circuit value problem to the problem of finding arbitrary solutions to SAC-graphs. The construction is adapted from Henglein's proof of P-hardness of left-linear semi-unification [13]. That proof, in turn, is adapted from Dwork, Kanellakis, and Mitchell's proof of P-hardness of unification [8].

Given a monotone circuit and an assignment to input gates, we construct an SAC-graph as follows. Each gate in the circuit yields a small "gadget" consisting of a few nodes and edges. Each gadget has a pair of designated nodes for every in- and outedge of the encoded gate:

1. Each input gate is represented by two $S$ nodes $n, n^{\prime}$. The output node pair is $\left(n, n^{\prime}\right)$.

2. Each and-gate is represented by three $S$ nodes $n, n^{\prime}, n^{\prime \prime}$. The two input node pairs are $\left(n, n^{\prime}\right)$ and $\left(n^{\prime}, n^{\prime \prime}\right)$. The output node pair is $\left(n, n^{\prime \prime}\right)$.

3. Each or-gate is represented by two $S$ nodes $n, n^{\prime}$. The two input node pairs are $\left(n, n^{\prime}\right)$ and $\left(n, n^{\prime}\right)$. The output node pair is also $\left(n, n^{\prime}\right)$.

4. Each fan-out gate is represented by two $N$ nodes $n, n^{\prime}$ and four $S$ nodes $n_{1}, n_{2}, n_{1}^{\prime}, n_{2}^{\prime}$, and four edges $n \stackrel{0}{\rightarrow} n_{1}, n \stackrel{1}{\rightarrow} n_{2}, n^{\prime} \stackrel{0}{\rightarrow} n_{1}^{\prime}$, and $n^{\prime} \stackrel{1}{\rightarrow} n_{2}^{\prime}$, where 0 indicates "left" and 1 indicates "right". The input node pair is $\left(n, n^{\prime}\right)$. The output node pairs are $\left(n_{1}, n_{1}^{\prime}\right)$ and $\left(n_{2}, n_{2}^{\prime}\right)$.

5. The output gate is represented by two $N$ nodes $n_{1}, n_{2}$ and one $S$ node $n_{2}^{\prime}$, and one edge $n_{2} \stackrel{l}{\rightarrow} n_{2}^{\prime}$. The input node pair is $\left(n_{1}, n_{2}\right)$.

Each edge in the circuit yields one or more edges in the SAC-graph, as follows. Suppose there is an edge from gate $g$ to gate $g^{\prime}$ in the circuit. We connect the corresponding output pair $\left(n, n^{\prime}\right)$ in the representation of $g$ to the corresponding input pair $\left(m, m^{\prime}\right)$ in the representation of $g^{\prime}$ by adding the edges $m \stackrel{\leq}{\rightarrow} n$ and $n^{\prime} \stackrel{\leq}{\rightrightarrows} m^{\prime}$.

Finally, consider an input gate which is assigned true by $a$. Suppose the gate is represented by $n, n^{\prime}$. We add the edge $n \leftrightarrows n^{\prime}$. 
Having now constructed the SAC-graph $G$, consider the two $N$ nodes $n_{1}, n_{2}$ in the representation of the output gate $g$. Clearly, the closure of $G$ contains an edge $n_{1} \stackrel{\leq}{\rightarrow} n_{2}$ if and only if $\bar{a}(g)=$ true. By Theorem 6.5 it follows, that $\bar{a}(g)=$ true if and only if $G$ is not solvable. Thus, finding arbitrary solutions to a SAC-system is P-hard.

In summary:

Theorem 8.1 All four type inference problems are P-complete.

\section{$9 \quad$ Examples}

We now give three examples of how the type inference algorithms work. We use the algorithm for the system with recursive types and subtyping.

The first example is taken from Section 2. Consider the object $o \equiv[l=$ $\varsigma(x) x . l]$. The expression o.l yields the following constraints and in turn the following graph:

$$
\begin{array}{ll}
o . l \quad & {\left[\begin{array}{l}
\llbracket o \rrbracket \leq[l:\langle o . l\rangle] \\
\langle o . l\rangle \leq \llbracket o . l \rrbracket
\end{array}\right.} \\
o \quad & {\left[\begin{array}{l}
{[l: \llbracket x . l \rrbracket] \leq \llbracket o \rrbracket} \\
x=[l: \llbracket x . l \rrbracket]
\end{array}\right.} \\
x . l \quad & {\left[\begin{array}{l}
\llbracket x \rrbracket \leq[l:\langle x . l\rangle] \\
\langle x . l\rangle \leq \llbracket x . l \rrbracket
\end{array}\right.} \\
x \quad & x \leq \llbracket x \rrbracket
\end{array}
$$

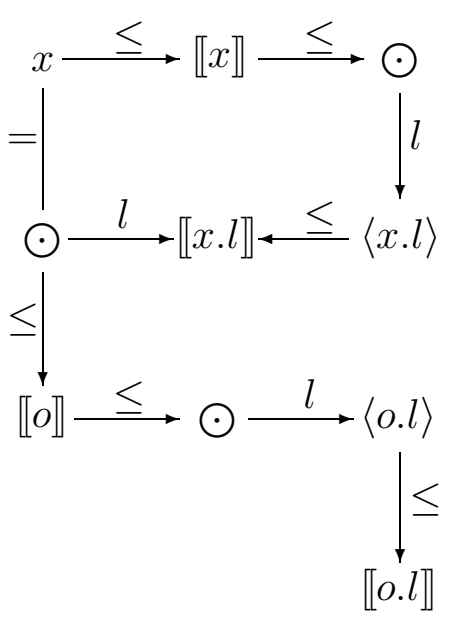

To the left of the constraints we have written from which expressions they are generated. In the graph we indicate $N$ nodes with the symbol $\odot$. To close the graph we only need to add three equality edges between the nodes $\llbracket x . l \rrbracket,\langle x . l\rangle$, and $\langle o . l\rangle$, and of course edges to make $\leq$ transitive. Clearly, the resulting graph is well-formed, hence solvable. By transforming the graph into an automaton, we get that the variable $x$ has the $\subseteq$-least annotation $[l:[]]$. 
The second example is also taken from Section 2. Consider the object $o^{\prime} \equiv[l=\varsigma(x) x]$. The expression $o^{\prime} . l$ yields the following constraints and in turn the following graph:

$$
\begin{aligned}
o^{\prime} . l & {\left[\begin{array}{l}
\llbracket o^{\prime} \rrbracket \leq\left[l:\left\langle o^{\prime} . l\right\rangle\right] \\
\left\langle o^{\prime} . l\right\rangle \leq \llbracket o^{\prime} . l \rrbracket
\end{array}\right.} \\
o^{\prime} \quad & {\left[\begin{array}{l}
{[l: \llbracket x \rrbracket] \leq \llbracket o^{\prime} \rrbracket} \\
x=[l: \llbracket x \rrbracket] \\
x
\end{array} \quad x \leq \llbracket x \rrbracket\right.}
\end{aligned}
$$

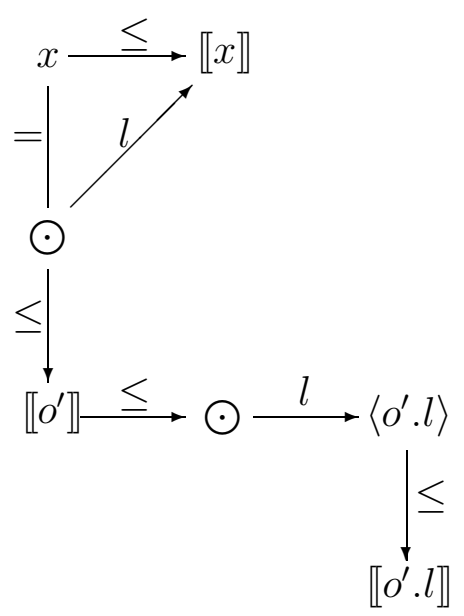

To close the graph we only need to add an equality edge between the nodes $\llbracket x \rrbracket$ and $\left\langle o^{\prime} . l\right\rangle$, and of course edges to make $\leq$ transitive. Clearly, the resulting graph is well-formed, hence solvable. By transforming the graph into an automaton, we get that the variable $x$ has the $\subseteq$-least annotation $[l:[]]$.

The third example is also taken from Section 2. Consider the object $o^{\prime \prime} \equiv[l=\varsigma(y) b]$, where $b \equiv y . l \Leftarrow \varsigma(x) x$. The expression $o^{\prime \prime} . l$ yields the following constraints and in turn the following graph: 


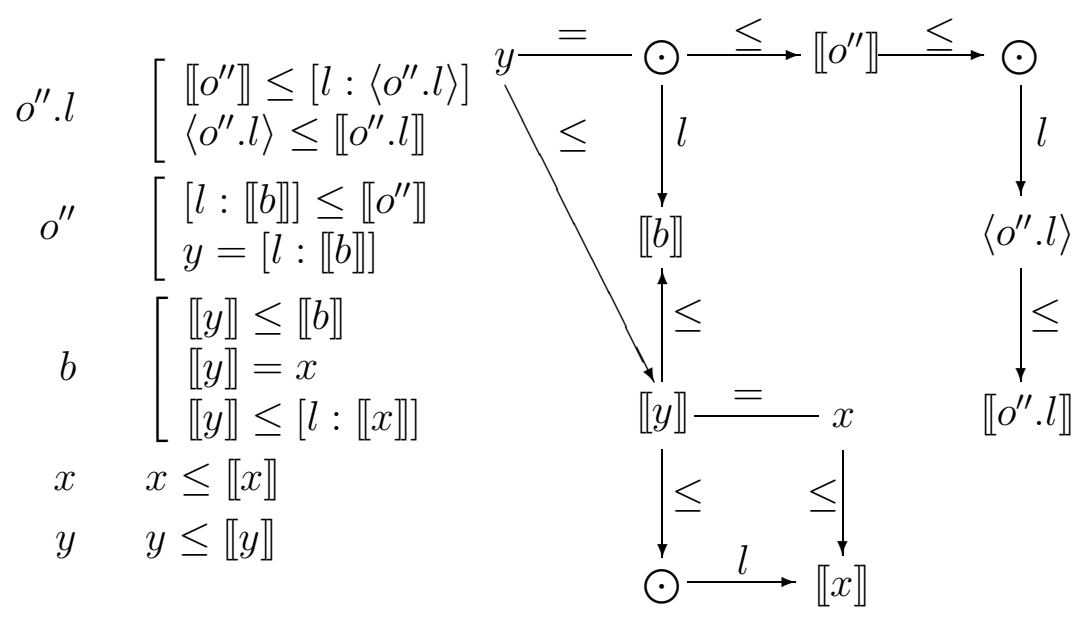

To close the graph we only need to add an equality edge between the nodes $\llbracket b \rrbracket$ and $\left\langle o^{\prime \prime} . l\right\rangle$, and of course edges to make $\leq$ transitive. Clearly, the resulting graph is well-formed, hence solvable. By transforming the graph into an automaton, we get that the variables $x, y$ both have the $\subseteq$-least annotation $[l:[]]$.

The fourth example illustrates what happens if the program is not typable. Consider the object $a \equiv[$ ]. The expression a.l yields the following constraints and in turn the following graph:

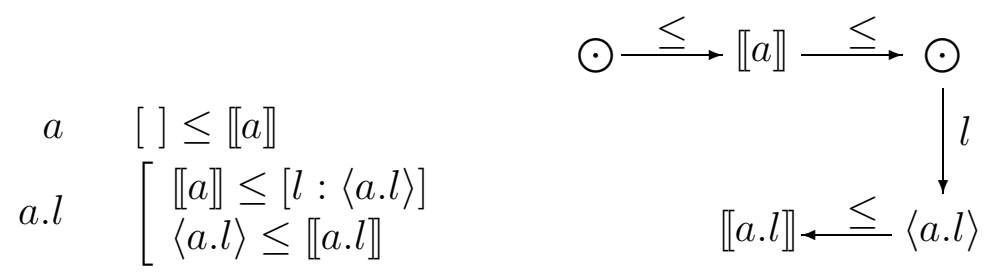

To close the graph we only need to add a single edge to make $\leq$ transitive. The resulting graph is not well-formed, however, because the leftmost node does not have an outgoing $l$ edge. Thus, the graph is not solvable. 


\section{Related work}

Type inference with subtyping has been studied for $\lambda$-calculi. In that setting, the problem of type inference with atomic subtyping can be presented as follows. Suppose types are finite trees over the binary function type constructor $\rightarrow$ and a partially ordered set $(\Sigma, \leq)$ of constants, that is, base types. The ordering on types is the smallest extension of $\leq$ such that $s \rightarrow t \leq s^{\prime} \rightarrow t^{\prime}$ if and only if $s^{\prime} \leq s$ and $t \leq t^{\prime}$. The type rules are those of simply typed $\lambda$-calculus with typed constants together with the subsumption rule. The type inference problem is to decide if a given $\lambda$-term is typable. This problem was introduced by Mitchell in 1984 [19] who showed that type inference is computable in NEXPTIME in the size of the program. If $(\Sigma, \leq)$ is a disjoint union of lattices, then type inference is computable in polynomial time [23]. Moreover, if $(\Sigma, \leq)$ is "tree-like", then type inference is computable in polynomial time [6]. In general, the type inference problem is $P S P A C E$ hard [14, 23]. Type inference with atomic subtyping has also been studied in combination with ML polymorphism [10, 11].

Objects do not have base types, so type inference with atomic subtyping does not apply to Abadi and Cardelli's calculus. The object types considered in this paper are rather like record types. Type inference for $\lambda$-calculi with records but no subtyping has been studied by Wand [24] and Remy [22]. In the presence of subtyping, unification-based approaches to type inference seem not to apply.

Type inference for $\lambda$-calculi with records and subtyping has been studied by Eifrig, Smith, and Trifonov [9], using the approach to type inference of Aiken and Wimmers [5]. Their algorithm does not immediately apply to Abadi and Cardelli's calculus because of the following difference between the subtyping relations. For records, the conventional subtyping relations makes every record type constructor covariant in all arguments. For example, $[l: A, m: C]$ is a subtype of $[l: B]$ provided that $A$ is a subtype of $B$. In Abadi and Cardelli's type system, $[l: A, m: C]$ can only be a subtype of $[l: B]$ if $A=B$. The conventional subtyping relation for records is unsound in the case of Abadi and Cardelli's calculus [2]. Even though the subtyping relation in Abadi and Cardelli's type system is smaller than the conventional one for record types, it yields a more complicated definition of closed ACgraphs, as illustrated in the following.

Our algorithms can easily be extended to handle also $\lambda$-terms and a con- 
travariant function space constructor, in outline as follows. The constraints for $\lambda$-terms are as usual (see for example [17]). The definition of AC-graph needs to be extended with a set $N_{\rightarrow}$ of nodes and a set $E_{\rightarrow}$ of edges. Each edge in $E_{\rightarrow}$ is labeled by either $L$ or $R$, where $L \notin \mathcal{N}$ and $R \notin \mathcal{N}$, where $\mathcal{N}$ is the set of method names. Each node in $N_{\rightarrow}$ corresponds to a function type constructor, and it has exactly two outgoing $E_{\rightarrow}$ edges, both to $S$ nodes: one edge labeled $L$ (indicating the left argument of $\rightarrow$ ) and one edge labeled $R$ (indicating the right argument of $\rightarrow$ ). The definition of closed AC-graph needs to be extended so that it is closed under the rule which says that the dashed edges exists whenever the solid ones do:

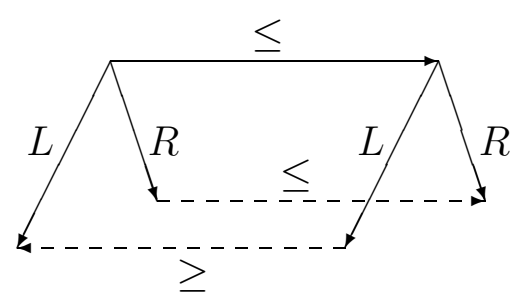

Clearly, closing such graphs can be done in $O\left(n^{3}\right)$ time. Finally, the notion of well-formed AC-graph needs to be restricted so that:

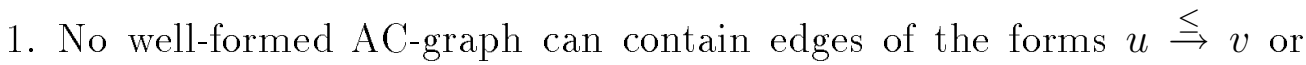
$v \stackrel{\leq}{\rightrightarrows} u$ where $u \in N$ and $v \in N_{\rightarrow}$,

2. No well-formed AC-graph can contain a pair of edges of the forms $u \leqq v$ and $u \stackrel{\leq}{\rightarrow} w$ where $u \in S, v \in N$, and $w \in N_{\rightarrow}$, and

3. No well-formed AC-graph can contain a pair of edges of the forms $v \stackrel{\leftrightarrows}{\Rightarrow} u$ and $w \stackrel{\leq}{\Rightarrow} u$ where $u \in S, v \in N$, and $w \in N \rightarrow$.

The extra checks can be done in $O\left(n^{2}\right)$ time where $n$ is the number of nodes. Given proper definitions of the new set of types and the new $\leq$ relation (along the lines of [17]), it should be possible to prove that Theorem 6.5 holds. For the program $\lambda(x)((x . a) x)$, the most powerful of the type inference algorithms infers that $x$ has the $\subseteq$-least annotation $[a:[] \rightarrow[]]$.

For a $\lambda$-calculus with records, our algorithm can be modified to handle the conventional subtyping relation for record types, simply by changing the definition of closed AC-graph such that if $u, v \in N, s, t \in S$, and the edges 
$u \stackrel{\text { ․ }}{\rightarrow}, u \stackrel{l}{\rightarrow} s, v \stackrel{l}{\rightarrow} t$ exist, then also $s \stackrel{\leqq}{\rightarrow} t$ exists. Clearly, closing a graph can be done in $O\left(n^{3}\right)$ time. The proof that Theorem 6.5 holds is left as an exercise for the reader.

In remains to be seen how to extend our algorithm to deal with ML polymorphism.

\section{Conclusion}

The type inference problems we have addressed are related to those treated by Eifrig, Smith, and Trifonov [9]. Specifically, our algorithm can be modified to handle functions and records, and it seems possible to modify their algorithm to handle the object calculus. The results of the two approaches appear to be complimentary. We have completeness results and efficient algorithms, while they have incremental algorithms that can handle ML polymorphism. Future work may attempt to combine the two approaches.

Acknowledgements. The author thanks Paris Kanellakis for comments that prompted me to find the reduction from AC-systems to the type inference problem. The author also thanks Fritz Henglein and Harry Mairson for discussions on proving P-hardness. Moreover, the author thanks Devdatt Dubhashi, Gudmund Frandsen, and Sven Skyum for discussions on algorithms for closing AC-graphs. Finally, the author thanks Ole Agesen and the anonymous referees for many helpful comments on a draft of the paper. A preliminary version of this paper appeared in Proc. LICS'94, Ninth Annual IEEE Symposium on Logic in Computer Science. The results of this paper were obtained while the author was at Northeastern University, Boston.

\section{References}

[1] Martín Abadi and Luca Cardelli. A semantics of object types. In Proc. LICS'94, Ninth Annual IEEE Symposium on Logic in Computer Science, pages $332-341,1994$.

[2] Martín Abadi and Luca Cardelli. A theory of primitive objects. Manuscript, February 1994. 
[3] Martín Abadi and Luca Cardelli. A theory of primitive objects: Second-order systems. In Proc. ESOP'94, European Symposium on Programming, pages 1-25. Springer-Verlag ( LNCS 788), 1994.

[4] Martín Abadi and Luca Cardelli. A theory of primitive objects: Untyped and first-order systems. In Proc. TACS'94, Theoretical Aspects of Computing Sofware, pages 296-320. Springer-Verlag (LNCS 789), 1994.

[5] Alexander Aiken and Edward Wimmers. Type inclusion constraints and type inference. In Proc. Conference on Functional Programming Languages and Computer Architecture, pages 31-41, 1993.

[6] Marcin Benke. Efficient type reconstruction in the presence of inheritance. Manuscript, 1994.

[7] Ravi B. Boppana and Michael Sipser. The complexity of finite functions. In J. van Leeuwen, A. Meyer, M. Nivat, M. Paterson, and D. Perrin, editors, Handbook of Theoretical Computer Science, volume A, chapter 14, pages 757804. Elsevier Science Publishers, Amsterdam; and MIT Press, 1990.

[8] C. Dwork, P. Kanellakis, and J. Mitchell. On the sequential nature of unification. Journal of Logic Programming, 1:35-50, 1984.

[9] J. Eifrig, S. Smith, and V. Trifonov. Type inference for recursively constrained types and it application to OOP. In Proc. Mathematical Foundations of Programming Semantics, 1995. To appear.

[10] You-Chin Fuh and Prateek Mishra. Type inference with subtypes. In Proc. ESOP'88, European Symposium on Programming, pages 94-114. SpringerVerlag ( $L N C S 300), 1988$.

[11] You-Chin Fuh and Prateek Mishra. Polymorphic subtype inference: Closing the theory-practice gap. In Proc. TAPSOFT'89, pages 167-183. SpringerVerlag ( LNCS 352), 1989.

[12] Fritz Henglein. Simple type inference and unification. Technical Report (SETL Newsletter) 232, Courant Institute of Mathematical Sciences, New York University, October 1988.

[13] Fritz Henglein. Fast left-linear semi-unification. In Proc. ICCI'90, International Conference on Computing and Information, pages 82-91. SpringerVerlag ( LNCS 468), 1990. 
[14] My Hoang and John C. Mitchell. Lower bounds on type inference with subtypes. In Proc. POPL'95, 22nd Annual SIGPLAN-SIGACT Symposium on Principles of Programming Languages, pages 176-185, 1995.

[15] G. F. Italiano. Amortized efficiency of a path retrieval data structure. Theoretical Computer Science, 48:273-281, 1986.

[16] Richard M. Karp and Vijaya Ramachandran. Parallel algorithms for sharedmemory machines. In J. van Leeuwen, A. Meyer, M. Nivat, M. Paterson, and D. Perrin, editors, Handbook of Theoretical Computer Science, volume A, chapter 17, pages 869-941. Elsevier Science Publishers, Amsterdam; and MIT Press, 1990.

[17] Dexter Kozen, Jens Palsberg, and Michael I. Schwartzbach. Efficient inference of partial types. Journal of Computer and System Sciences, 49(2):306-324, 1994. Also in Proc. FOCS'92, 33rd IEEE Symposium on Foundations of Computer Science, pages 363-371, Pittsburgh, Pennsylvania, October 1992.

[18] Bent B. Kristensen, Ole Lehrmann Madsen, Birger Møller-Pedersen, and Kristen Nygaard. The BETA programming language. In Bruce Shriver and Peter Wegner, editors, Research Directions in Object-Oriented Programming, pages 7-48. MIT Press, 1987.

[19] John Mitchell. Coercion and type inference. In Eleventh Symposium on Principles of Programming Languages, pages 175-185, 1984.

[20] John C. Mitchell, Furio Honsell, and Kathleen Fisher. A lambda calculus of objects and method specialization. In LICS'93, Eighth Annual Symposium on Logic in Computer Science, pages 26-38, 1993.

[21] J. A. La Poutré and J. van Leeuwen. Maintenance of transitive closure and transitive reduction of graphs. In Proc. Workshop on Graph-Theoretic Concepts in Computer Science, pages 106-120. Springer-Verlag (LNCS 314), 1988.

[22] Didier Rémy. Typechecking records and variants in a natural extension of ML. In Sixteenth Symposium on Principles of Programming Languages, pages 7788, 1989 .

[23] Jerzy Tiuryn. Subtype inequalities. In LICS'92, Seventh Annual IEEE Symposium on Logic in Computer Science, pages 308-315, 1992.

[24] Mitchell Wand. Type inference for record concatenation and multiple inheritance. In LICS'89, Fourth Annual Symposium on Logic in Computer Science, pages $92-97,1989$. 


\section{Recent Publications in the BRICS Report Series}

RS-95-32 Jens Palsberg. Efficient Inference of Object Types. June 1995. 32 pp. To appear in Information and Computation. Preliminary version appears in Ninth Annual IEEE Symposium on Logic in Computer Science, LICS '94 Proceedings, pages 186-195.

RS-95-31 Jens Palsberg and Peter Ørbæk. Trust in the $\lambda$-calculus. June 1995. 32 pp. To appear in Static Analysis: 2nd International Symposium, SAS '95 Proceedings, 1995.

RS-95-30 Franck van Breugel. From Branching to Linear Metric Domains (and back). June 1995. 30 pp. Abstract appeared in Engberg, Larsen, and Mosses, editors, 6th Nordic Workshop on Programming Theory, NWPT '6 Proceedings, 1994, pages 444-447.

RS-95-29 Nils Klarlund. An $n \log n$ Algorithm for Online BDD Refinement. May 1995. 20 pp.

RS-95-28 Luca Aceto and Jan Friso Groote. A Complete Equational Axiomatization for MPA with String Iteration. May 1995. 39 pp.

RS-95-27 David Janin and Igor Walukiewicz. Automata for the $\mu$ calculus and Related Results. May 1995. 11 pp. To appear in Mathematical Foundations of Computer Science: 20th Int. Symposium, MFCS '95 Proceedings, LNCS, 1995.

RS-95-26 Faith Fich and Peter Bro Miltersen. Tables should be sorted (on random access machines). May 1995. 11 pp. To appear in Algorithms and Data Structures: 4th Workshop, WADS '95 Proceedings, LNCS, 1995.

RS-95-25 Søren B. Lassen. Basic Action Theory. May 1995. 47 pp.

RS-95-24 Peter Ørbæk. Can you Trust your Data? April 1995. 15 pp. Appears in Mosses, Nielsen, and Schwartzbach, editors, Theory and Practice of Software Development. 6th International Joint Conference CAAP/FASE, TAPSOFT ' 95 Proceedings, LNCS 915, 1995, pages 575-590.

RS-95-23 Allan Cheng and Mogens Nielsen. Open Maps (at) Work. April 1995. 33 pp. 